\title{
Una experiencia encarnada de investigación escénica en estudios teatrales universitarios
}

\author{
An embodied experience of performing arts research in university \\ theater studies
}

Carolina MARTÍNEZ-LÓPEZ. Universitat de Girona (España). carolina.martinez@eram.cat

Resumen. Este artículo presenta una propuesta pedagógica y de investigación en estudios teatrales universitarios basada en el análisis teórico y en la experiencia física, intelectual y emocional de procesos creativos escénicos. A lo largo del mismo, veremos cómo la dificultad de los estudios teatrales para integrarse en el terreno científico y en la academia puede utilizarse para construir nuevas vías docentes y creativas, atendiendo a la especificidad del teatro, vivo y efímero por naturaleza. Transitaremos para ello el camino de la Investigación Basada en las Artes, la A/r/ tografía (rama de la IBA) y las experiencias previas de Investigación Basada en el Teatro. Aunaremos una visión organicista y holística basada en la idea del arte como experiencia encarnada, y la aproximación a la investigación como un trabajo en proceso permanente. En cuanto a lo estético, primará una percepción transversal de lo escénico en el marco de las denominadas "teatralidades expandidas" ligadas al concepto de "performativo". En una segunda fase, enlazaremos esta teoría con la descripción y el análisis de casos prácticos llevados a cabo en el Grado de Artes Escénicas de la Universitat de Girona (EU ERAM), para intentar valorar la eficacia de la propuesta, además de buscar ir asentando unos parámetros válidos para su evaluación.

Palabras clave: estudios universitarios de Artes Escénicas, Investigación Basada en las Artes, experiencia encarnada, A/r/tografía, performativo

\footnotetext{
Abstract. This article presents a pedagogical and research proposal in Performing Arts university studies based on theoretical analysis, and on physical, intellectual and emotional experience of scenic creative processes. Throughout it, we will see
} 
how the difficulty of Performing Arts studies to integrate into the scientific field and into the Academy can be used to build new educational and creative paths, taking into account the specificity of Theater, alive and ephemeral by nature. We will go through the path of Arts-Based Research, A / r / tography (branch of ABR) and previous experiences of Theater-Based Research. We will combine an organic and holistic vision based on the idea of art as an embodied experience, and the approach to research as a work in permanent process. As for the aesthetic, a transversal perception of theatre will prevail within the framework of the so-called "expanded theatricalities" linked to the concept of "performative". In a second phase, we will link this theory with the description and analysis of practical cases carried out in the Degree of Performing Arts of the University of Girona (EU ERAM), to try to assess the effectiveness of the proposal, in addition to seeking to settle valid parameters for evaluation.

Keywords: Performing Arts university studies, Art-based Research, embodied experience, $\mathrm{A} / \mathrm{r} /$ tography, performative

\section{Punto de partida}

La integración de los estudios artísticos en la academia implica un especial grado complejidad por tratarse de disciplinas cuyo contenido debe ser práctico, técnico y creativo, pero también teórico y científico. En España, y en concreto en el caso de las artes escénicas, debido a las características actuales de los sistemas de evaluación tanto de docentes como de los estudios de grado y de máster por parte de la Agencia Nacional de Evaluación de la Calidad y Acreditación (ANECA), conlleva además el hecho de tener que hacer determinadas concesiones en cuanto al currículo y al profesorado, lo que explica la actual coexistencia en nuestro país de grados superiores no universitarios en escuelas de arte dramático y conservatorios, y de grados universitarios que, si bien tienen un largo recorrido en otros países europeos, en Estados Unidos y Latinoamérica ${ }^{1}$, en España son muy recientes (el primero se aprueba en $2008^{2}$ ).

Apuntada la complejidad y la problemática de los estudios teatrales universitarios en nuestro país para integrarse en un sistema educativo regido principalmente por un tipo de docencia e investigación científicas, veremos en este artículo cómo

\footnotetext{
1. En Francia, la implantación de los estudios teatrales en la Universidad se produce hacia el final de los años ' 60 del siglo XX, cuando se separan de la carrera de letras, siendo la Universidad París 8 con su sede experimental de Vincennes una de las pioneras; en Argentina la creación de las primeras licenciaturas en teatro se remonta a los años ' 80 del siglo XX; en Estados Unidos, la emblemática Tisch School of the Arts ofrece desde el '74 un Grado en Drama, y abre en el '80 la cátedra de Performance Studies, de la mano de Richard Schechner.
}

2. Universidad Antonio de Nebrija. Universidad Europea de Madrid y Universidad Rey Juan Carlos (2010). El Grado de Artes Escénicas de la Universitat de Girona (EU ERAM) se aprueba en 2014. Los primeros Masters oficiales se aprueban en 2009. [Datos: programa Verifica (ANECA)]. 
aprovechar dichas dificultades para intentar construir nuevas vías pedagógicas, de investigación y creativas, utilizando el entorno de la universidad, que puede ser perfecto para ello, y transitando el camino de la IBA (Investigación Basada en las Artes) o ABR (Arts-Based Research).

La IBA, aunque todavía en proceso de legitimación por parte de algunos sectores de la academia, se ha mostrado en las últimas décadas como una herramienta, que aúna arte e investigación, ideal para ser aplicada a la enseñanza. Si la investigación basada en las artes aplicada a la educación (Arts-Based Educational ResearchABER) defiende un tipo de investigación en constante transformación y en un perpetuo devenir (Baron y Eisner, 2011), el teatro - experiencia viva (Brook, 2015)se muestra como un arte idóneo para ponerla en práctica, ya que tanto si trabajamos con material documental, autobiográfico o ficción, los procesos escénicos y las relaciones que en ellos se producen son siempre reflejos de la realidad y están penetrados por ella. Toda pedagogía artística tiene una especificidad y en el caso de las artes escénicas esta está ligada a lo experiencial y al cuerpo, lo que hace necesaria la apertura de nuevos procesos de aprendizaje. Merleau-Ponty (2017) critica los métodos científicos por su impotencia para hacer frente a la filosofía, el arte y la experiencia del cuerpo, afirmando que, puesto que "somos el compuesto de alma y cuerpo" (p. 45) tiene que existir un pensamiento sobre ello. Añade, además, que la visión "nace 'con ocasión' de lo que acaece en el cuerpo, es 'excitada' a pensar por el cuerpo" (p. 42), algo que comparte Fischer-Lichte (2017) en relación a la vivencia y la aprehensión de lo escénico. En la misma línea hablaba Dewey (2008) del arte como experiencia organicista y holística -y en el caso de lo escénico podemos decir casi carnal-, defendiendo una teoría estética a la contra de las teorías hegemónicas sobre el arte. De Dewey (2008) nos interesa también su distinción del doble carácter del arte: autónomo y específico por un lado, y por otro relacional.

\section{Objetivos de la investigación, hipótesis y metodología}

Después de contextualizar y analizar las posibilidades del uso de la investigación basada en las artes y en el teatro, este artículo abordará, con una perspectiva cercana a la $\mathrm{A} / \mathrm{r} /$ tografía, desde la teoría y desde mi experiencia práctica a lo largo de cuatro años como profesora en el Grado de Artes Escénicas de la Escuela Universitaria ERAM de la Universitat de Girona, cómo se puede aplicar la investigación basada en las artes a la enseñanza superior de este campo, en este caso, de asignaturas teóricas del plan de estudios pero que, debido a su especificidad, se abordan y se enseñan también desde lo práctico. El tipo de investigación aquí aplicada tiene como pilar su carácter experiencial [ligado al concepto de lo performativo de FischerLichte (2017)] y, desde el punto de vista de lo estético, estará en relación con una percepción liminal de lo escénico, en el marco de las denominadas, desde hace una década por el teórico José A. Sánchez, "teatralidades expandidas". 
Aunando la teoría sobre la IBA, la investigación aplicada basada en lo escénico y la idea del arte como experiencia, y el análisis de los casos experimentados, se intentará valorar la eficacia de la aplicación pedagógica de procesos creativos escénicos expandidos, además de intentar ir asentando unos parámetros válidos para su evaluación. La propuesta que aquí presentaremos tiene que ver con la experiencia física, intelectual y emocional de procesos artísticos como forma de aprendizaje de la creación, la interpretación y la teoría escénica, y desde una perspectiva a/r/ tográfica.

Si la IBA contempla la "investigación como puzle" (Hernández, 2011, p. 112), nuestro artículo también será una especie de puzle que irá encajando unas piezas con otras construyendo progresivamente un discurso, pero a la vez dejando resquicios para que se manifieste aquello del proceso que no se puede verbalizar, creando una posibilidad de lectura poliédrica.

\section{Justificación del uso de la IBA en los estudios teatrales universitarios}

Si hasta hace poco tiempo se aplicaban los mismos criterios para la investigación científica que para la artística, sin reparar en las particularidades de esta última, la IBA nace con el objetivo de crear y legitimar un contexto y unos parámetros correspondientes a la particularidad del campo del arte, que, como proceso de conocimiento que no es lineal no se puede limitar, como tampoco sus procesos de investigación y de aprendizaje. Esta perspectiva permite la aceptación de la obra artística en sí y de sus procesos como resultados de la investigación (Della Fonte et al., 2015), siendo cada vez más frecuente la presentación de tesis doctorales o de trabajos de investigación que incluyen la obra artística y sus procesos como resultado de la misma. Podemos añadir que, a partir del siglo XX, el arte va siempre vinculado a la investigación (Sánchez y Pérez Royo, 2010), es investigación; de modo que "la práctica artística puede ser considerada investigación si su propósito es aumentar nuestro conocimiento y comprensión, mediante una investigación original [...] haciendo preguntas pertinentes en el contexto investigador y en el mundo del arte" (Borgdorff, 2012, p. 43), difundiéndose tanto los procesos como los resultados.

La ABR nace tras la crisis del positivismo y el cientifismo como parte del giro narrativo en las ciencias sociales -en cuanto a "formas narrativas que representen geografías de la experiencia humana" (Hernández, 2008, p. 89)-, y por influencia de la perspectiva feminista, con el fin de vincular la investigación y las artes. Para Eisner (2004), es clara la contribución de las artes al desarrollo cognitivo, siendo necesario incorporarlas como objeto y como medio de conocimiento y reflexión, diferenciadas de las llamadas "manualidades", por generar significados nuevos diferentes de los generados por lo verbal. Conjuntamente con Barone (cit. en Hernández, 2008), Eisner sostendrá que "la investigación científica es solo un tipo de investigación, pero no es la única forma de investigación posible, sobre todo 
si se trata de investigar fenómenos relacionados con comportamientos humanos, relaciones sociales o representaciones simbólicas" (p. 89). Ya hemos mencionado también en la introducción las propuestas alternativas al acceso y la generación de conocimiento de Dewey y de Merleau-Ponty.

Leavy (2018a) define la IBA como una herramienta transdisciplinar, "intersección entre el arte y la ciencia” (p. 3), áreas hasta hace poco tiempo presentadas como opuestas, pero que en realidad tienen muchas cosas en común, ya que ambas "exploran, iluminan y representan aspectos de la vida humana y de los mundos natural y social de los que somos parte" (p. 3).

Son muchas y matizadas las definiciones de la ABR, como señalan Leavy (2018a) y Hernández(2008), pero convergen en loesencial: todas ellas giran en tornoa la empatía, a múltiples maneras de conocimiento y reflexionan sobre la complejidad humana, atendiendo a una realidad prismática, aunando artey estética, buscando "otras maneras de mirar y representar la experiencia" y tratando "de desvelar aquello de lo que no se habla" (Hernández, 2008, p. 94). Todas ellas compartirían además con Dewey (2008) y con Bourriaud (2015), el punto de vista relacional del arte. Para Vidiella (2018), "este marco implica un giro importante hacia una aproximación no representacional de la realidad [...] sino como creación (performativa) de la misma" (p. 62).

Como ya hemos comentado, tanto el campo de la IBA como su aplicación a la educación, son áreas aún por definirse y por consolidarse y legitimarse, entrañando en ocasiones confusiones en sus límites. Leavy (2018a) intenta acotar las ventajas de la ABR, afirmando que permite llegar a un conocimiento de otro modo inaccesible, forjar micro y macro conexiones, trabajar y producir una visión holística y multi y transdisciplinar, evocativa y provocativa; contribuye a luchar contra estereotipos e ideologías dominantes, a crear conciencia crítica, empatía, múltiples significados, y a fomentar prácticas participatorias con utilidad pública y pedagógica. También intenta definir las condiciones para poder trabajar desde la perspectiva ABR: "flexibilidad, apertura, intuición"; "pensar conceptualmente, simbólicamente, metafóricamente"; "ética de valores"; "pensar como un artista"; y pensar como un "intelectual público" (Leavy, 2018a, pp. 11-12)

\section{Posibles caminos: la Investigación Basada en el Teatro y la A/r/tografía}

Dentro de la IBA se contempla la rama de Investigación Basada en el Teatro. Estas experiencias previas han sido clasificadas y enmarcadas en un espectro de categorías por Beck, Belliveau, Lea y Wager (2011) $)^{3}$ y alimentan especialmente un

3. Algunos de los términos que recopilan son verbatim theatre (Paget, 1997), performance/performanced ethnography (Denzin, 1997), documentary theatre (Dawson, 1999), y ethnodrama/ethnotheatre (Saldaña, 2003) (Beck, Belliveau, Lea \& Wager (2011). 
tipo de investigación sociológica, etnográfica e incluso histórica. La más conocida de ellas es la denominada "etnodrama", un formato que mezcla preocupaciones sociales y estéticas, y cuya denominación fue instaurada y acuñada por Saldaña (2011), defendiendo especialmente la escena como un lugar en el que se reproducen contextos, comportamientos y relaciones similares a los de la realidad, en la línea de lo que Boal (2009) defendía con su teatro del oprimido, para poder trabajar las relaciones de poder, lo autobiográfico, el trauma, las conexiones interpersonales, etc. en un entorno "ficticio", paralelo al real.

Nuestra propuestase aleja de la mayoría de experiencias previas pordiferentes razones. En primer lugar, los protagonistas de las experiencias son estudiantes universitarios y futuros profesionales en este campo, y no grupos de estudio, practicantes amateurs o estudiantes de primaria ${ }^{5}$ o secundaria. En segundo lugar, no trabajan directamente con material autobiográfico ni con fines directos terapéuticos, aunque sí con su experiencia personal como sujetos inmersos en un proceso creativo que dará lugar a un objeto indivisible de sus sujetos creadores (Sánchez, 2010), su subjetividad, circunstancias y contextos. Otra diferencia estribaría en que la investigación basada en el teatro en general tiene la mayoría de las veces como instrumento y producto la escritura o el guion [Belliveau y Lea (Eds.), 2016], mientras que el enfoque de nuestros estudios es más amplio en cuanto a lo escénico y no pasa necesariamente por lo verbal o lo textual, como veremos más adelante. Beck et al. (2011) distinguen entre el uso del teatro para dar forma y presentar la investigación, es decir, como técnica para recopilar datos y para construir la investigación, y la práctica teatral. Para nosotros, desde nuestro planteamiento, no existe una práctica escénica posible sin investigación, por lo que, en nuestro caso, utilizamos las herramientas formales escénicas para presentar los resultados de la investigación, al mismo tiempo que esta se ha ido tejiendo gracias a procesos de creación escénicos de investigación. Los autores citados achacan además una diferencia existente en cuanto a los procesos que se utilizan en la investigación basada en lo escénico y los procesos reales de creación escénica, mientras que las experiencias llevadas a cabo en el Grado de Artes Escénicas de la UdG se dirigen precisamente a que los estudiantes trabajen un proceso creativo similar al desarrollado por los artistas escénicos, aunque el formato final de presentación tenga más que ver con el de un work-in-progress que con el de un espectáculo cerrado, y tenga por audiencia a sus propios compañeros. Una última característica de la investigación basada en el teatro que la aleja de la perspectiva aquí propuesta es que separan danza, teatro y performance (entendida en este caso como "arte de acción").

\footnotetext{
4. Un etnodrama es "una obra escrita consistente en una significativa selección dramatizada de narrativas recogidas de transcripciones de entrevistas, notas de campo de observación participativa, entradas de diario, memorias/experiencias personales, y/o artefactos impresos o virtuales" (Saldaña, 2011, p. 13).

5. En este sentido cabe destacar la experiencia testimoniada en Saz, I. (2018). Ser (una isla). Teatralidades expandidas en la escuela. Cuenca: Ediciones de la Universidad de Castilla-La Mancha, enmarcada en el proyecto de investigación "Teatralidades disidentes" del Ministerio de Economía, Industria y Competitividad.
} 
La perspectiva performativa señalada por Hernández (2008), aunque interesante, se centra especialmente en el papel del cuerpo en la narrativa autoetnográfica, mientras que nuestro enfoque de lo performativo -término demasiado polisémico y hasta vago desde Austin (2016) - y de la corporeidad tiene más que ver con las definiciones de Fischer-Lichte (2017). Si bien, concuerda con Hernández (2008) en la necesidad de aprender desde la subjetividad de cada uno, desde la experiencia propia, asentándose sobre la idea base de Berger (2017), de que "(...) lo que sabemos o lo que creemos afecta a cómo vemos las cosas" (p. 8).

Teniendo en cuenta estas experiencias previas, el camino más adecuado resultó buscar una vía nueva acorde a una concepción más amplia del arte escénico y a su ligazón intrínseca a lo corporal y lo experiencial. La A/r/tografíase mostró, en este sentido, como una herramienta muy útil.

Según la definen Irwin \& Springgay (cit. en Irwin, et al., 2018), la A/r/tografía es una metodología de investigación basada en la práctica en la cual "las tres maneras de entender la experiencia -teoría, práctica, y poiesis - van juntos y forman maneras rizomáticas de experienciar el mundo" (p. 43). Las tres primeras letras de $\mathrm{A} / \mathrm{r} /$ tografía crean un juego de palabras con las iniciales de esa figura triangular que contendría al artista (artist) -y aquí a artista podríamos añadirle el matiz que propone Eisner (2004) del profesor como artista-, al investigador (researcher) y al profesor (teacher); implicando una función también triangular: hacer, aprender y saber (Irwin et al., 2018). Esta es la figura desde la cual yo, como profesora, investigadora y artista, he intentado guiar el aprendizaje en las asignaturas que se concretarán más adelante, realizando una retroalimentación entre actividad artística e investigadora y pedagógica, permitiendo en lugar de descubrir lo que ya existe, abrazar cada nueva idea como una nueva realidad, creando un contexto de práctica abierta, en proceso, en "continuo devenir" (Irwin, et al., 2018, p. 49) y desarrollando nuevas maneras de aprender y entender. Igual que el artista trabaja para hacer visible lo invisible, la $\mathrm{A} / \mathrm{r} /$ tografía nos permite experienciar aquello que aún es desconocido, y la manera de comprometernos es confiar en el proceso artístico ya que nos adentramos en él sin un final predeterminado, viviendo dentro de la práctica (Irwin et al., 2018). Lea, Belliveau, Wager, y Beck (2011), aunque de nuevo con un contexto social y unos objetivos diferentes de los nuestros, remarcan la interesante conexión entre la investigación basada en el teatro y la $\mathrm{A} / \mathrm{r} /$ tografía, defendiendo que en un proceso creativo escénico se dan las seis características del proceso a/r/tográfico: "contigüidad, investigación viva, metáfora y metonimia, aperturas, reverberaciones y excesos" (Lea, et al., 2011); elementos que estarán presentes en nuestra experiencia y que veremos concretarse después. 


\section{La experiencia de procesos de investigación y creación escénica como herramienta pedagógica universitaria}

Un nuevo enfoque desde lo experiencial y lo performativo. Creando el entorno para la formación de intérpretes-creadores-investigadores

El Grado de Artes Escénicas de la Universitat de Girona (EU ERAM), es práctico en sus dos terceras partes, y en él se busca que los estudiantes tengan un aprendizaje global del campo de las artes escénicas y no solo centrado en la interpretación. De este planteamiento nace la necesidad de impartir las asignaturas teóricas y enfocar los trabajos de fin de grado desde una perspectiva que permita ligar la teoría con la práctica y que desarrolle un aprendizaje experiencial.

Para Fischer-Lichte (2017), el nuevo carácter de lo escénico, cuya aprehensión no puede ser sino experiencial, ya no permite ser abordado desde la estética tradicional o la semiótica, sino creando una nueva teoría que ella denomina "estética de lo performativo" y que se caracteriza por una aproximación a lo escénico como acontecimiento donde no hay frontera entre los roles, y donde importa más la experiencia que el significado. La experiencia primero se produce en el cuerpo del espectador por las acciones (autorreferenciales) propuestas por el actor/ creador, y después son procesadas e interpretadas por la mente. Esta complejidad, multidisciplinariedad y especificidad de las artes escénicas contemporáneas, hacen necesario un nuevo enfoque investigador y pedagógico en este campo.

Ya apuntábamos al principio del texto que es imposible en el siglo XXI desligar arte e investigación, y la complejidad de la relación entre ambos se deriva muchas veces de la diferente percepción de esos conceptos para cada uno de los participantes en el proceso, entre ellos la universidad, siendo además cada vez más difusos los límites entre práctica y teoría, con el riesgo de olvidar la reflexión. La aplicación de la IBA en el medio universitario se nos muestra como un medio ideal para integrar teoría, práctica e investigación, y también para acabar con la brecha entre la academia y el arte, y con la idea de genio (Sánchez y Pérez Royo, 2010). Asimismo, podemos decir que permite sacar el arte de los museos, recuperando su función original como herramienta para compensar las carencias de la mente humana (De Botton y Armstrong, 2017). Los estudios universitarios de artes escénicas no pueden enseñar a ser buenos actores, directores, dramaturgos o creadores, pero sí pueden crear el terreno para que los estudiantes transiten este camino, para que lleguen a esos espacios liminales y a esos intersticios donde el arte aparece, enseñando algo más que técnica. También pueden ayudar a crear espacios de encuentro y de interconexión en un terreno gobernado por la tautología irrefutable del actor de trabajar con su propio cuerpo. Nosotros no somos artistas formadores de artistas, somos artistas/investigadores/docentes que compartimos/ofrecemos un proceso de aprendizaje a estudiantes/investigadores/creadores. 
La idea de las experiencias que se describirán en el apartado siguiente parte de desplegar ante los estudiantes diferentes procesos creativos escénicos en los que priman la multidisciplinariedad y transdisciplinariedad características de la IBA y de la percepción de las teatralidades expandidas y de lo performativo que aquí se defienden, basados en procesos creativos que yo he tenido la oportunidad de experimentar. Son procesos que luchan por la horizontalidad, la búsqueda y el diálogo con el espectador, en pro de un lenguaje escénico autónomo y en sintonía con las prácticas participativas que ya empezaron a encarnar compañías como The Living Theatre, el Odin Teatret o Peter Brook, que contemplan el teatro como experiencia transformadora. Mediante la perspectiva a/r/tográfica incorporamos "el texto, lo visual y/o lo artístico para desafiar, provocar y frustrar el deseo de un significado único, final, estable o fijo" (Irwin et al., 2018, p. 50).

\section{Descripción de experiencias prácticas}

Las prácticas descritas a continuación se basan en la defensa de Sullivan (2004) de que el proceso investigador de la práctica artística puede tener lugar en la experiencia del taller, y tienen como fin proponer a los estudiantes sumergirse en procesos artísticos para conseguir algunas de las competencias de aprendizaje marcadas en las asignaturas. Como señalábamos anteriormente, aunque estas son de carácter teórico, consideramos indispensable una aproximación desde la práctica. Además de este marco general todos los ejercicios comparten una serie de características:

-A diferencia de propuestas previas sobre investigación basada en el teatro, no tienen el texto como eje principal de las creaciones, contemplando la escena como una globalidad estética cuyo marco teórico se basa principalmente en los conceptos de posdramático (Lehmann, 2013) y de performativo (Lichte, 2017). En el caso de que se utilice el texto, se iría mucho más allá de las clasificaciones de textos performativos propuestas por Denzin (en Sanders, 2006), buscando desarrollar un tipo de investigación y creación ligadas a lo experiencial, más que a lo narrativo.

-Se acompañan y retroalimentan de una parte escrita $\llcorner$ conectada a la práctica mediante la fluidez- en la que, tras crear un contexto teórico y de referencias, explican cómo ha sido el proceso creativo, acabando con una autoevaluación escrita.

-Desde la perspectiva a/r/tográfica, yo como creadora, investigadora y docente, ofrezco un contexto y la vivencia de un proceso en el que los/las estudiantes deben buscar la horizontalidad y la autorregulación del grupo.

-El alumnado distribuye los roles en la dirección, la escenografía, la dramaturgia, la interpretación, etc. pero todo el grupo participa del proceso de negociación y la toma de decisiones. Todos los ejercicios funcionan con el formato de creación colectiva en el que "los participantes son tanto investigadores como los investigados que presentarán públicamente su investigación” (Norris, 2018, p. 298). 
-Parten de procesos en los que se diluyen las fronteras y los roles actor/espectador, de modo que en el aula se invertirán también en algunos momentos los roles profesor/ actor/director y estudiantes/espectadores.

-Están basados en el concepto de translation, traducción o transpolación (Chilton en Leavy, 2018b), mediante el cual se proponen prácticas consistentes en trasladar un contenido de una forma a otra y/o de un dispositivo a otro.

-Siguen las premisas de creadores y teóricos que, desde el siglo XX lucharon por una concepción escénica global, empezando por Wagner y su Gesamtkunstwerk u obra de arte total, y la defensa de lo dionisíaco de Nietzsche (2012), alimentados más tarde por la irrupción de las vanguardias históricas y sus propuestas escénicas, que contribuirán a la creación de la escena contemporánea tal como la conocemos hoy en día (Sánchez, 1999). Es por ello que la aproximación del artículo, en muchos momentos, puede resultar más cercana a las artes visuales que a lo teatral.

-Se alienta el uso de la multidisciplinariedad y de la metáfora, de modo que el proceso y la forma que vaya apareciendo tenga una relación con el contenido.

-Se presta una atención especial al proceso.

-Al acabar el proceso de creación los ejercicios se presentan, a modo de work-inprogress, ante el resto de estudiantes para crear un espacio abierto al diálogo y el encuentro.

A continuación se explicará un ejercicio clave de cada una de las asignaturas en las que se han llevado a cabo estas prácticas:

-"Del libro a la pantalla. Realización de un booktrailer" se encuadra dentro de la asignatura de primer curso Literatura Dramática I (GAE1). Hay siete textos básicos de lectura obligatoria, por lo que se crean siete grupos, quedando en cada uno una media aproximada de seis alumnos (nuestra ratio media es de 45 estudiantes por curso) que adoptarán los diferentes roles necesarios en un pequeño rodaje. La primera parte del proceso consiste en una inmersión total por parte de los/las estudiantes en el texto, sin la cual sería imposible poder hacer la traslación de los conflictos y las ideas principales de la obra al terreno audiovisual. Cabe señalar que la demanda del ejercicio no consiste en conseguir un grado de perfección técnica, sino en saber gestionar y distribuir el proceso en el grupo, negociar decisiones y observar la poliedricidad que tiene un texto dramático clásico a la hora de poder ser abordado. El apoyo técnico viene de los estudiantes del Grado en Comunicación Audiovisual y Multimedia de nuestro centro. El objetivo del ejercicio es aproximarse a un texto dramático clásico desde otro lugar, siendo capaces de traducirlo en imágenes sin necesidad, aprovechando las ventajas que la tecnología y su democratización nos 
ofrecen para crear "espacios en los que pensar y sentir, y conocer y entender el mundo de manera diferente y tan válida como la de la palabra escrita" (Hearing y Jones, 2018, p. 435).

-“Actualización de una escena dramática clásica”. Ejercicio propuesto en Literatura Dramática II (GAE1). La distribución de los grupos es similar a la anterior, al igual que el trabajo previo de análisis e inmersión en el texto. Las pautas para el trabajo tienen que ver con el traslado a nuestra época y nuestra cultura de una escena, su contexto, sus personajes y su conflicto para poder entender de primera mano la universalidad y vigencia del mismo. Tras el estudio del texto, los/las estudiantes hacen una reescritura de la escena con estas pautas. En este sentido, podría ser el que más se aproximase al "etnodrama", ya que en el proceso, aparecen indefectiblemente características relacionadas con el contexto y las circunstancias de los participantes. En una segunda fase se realiza una puesta en escena de la escena reescrita.

-La asignatura Teorías Dramáticas II (GAE2) gira especialmente en torno a la creación escénica del siglo XX que supera la crisis del drama burgués del XIX (Szondi, 2011), sentando las bases para el teatro contemporáneo y actual. El ejercicio consiste en la creación de una pequeña puesta en escena a partir de la obra de un/a creador/a, tras haber realizado una pequeña investigación teórica con pautas científicas que les permitirá realizar la parte práctica, en la que harán una pequeña creación escénica propia basada en el artista elegido. Los artistas escénicos objeto del ejercicio son artistas de los siglos XX y XXI cuyo legado permite abrirse a multitud de significados que la mayoría de las veces escapan a una lectura racional o intelectual, primando muchas veces la intuición (Artaud, 2011; Chéjov, 1999), el azar (John Cage), lo fragmentario (Pina Bausch), o el extrañamiento y casi rechazo por parte del espectador (Marina Abramovic, Jan Fabre). Se sumergen así en procesos creativos que privilegian la apertura, la incertidumbre y la búsqueda para llegar a un tipo de conocimiento nuevo, experimentando y creando desde dentro del universo creativo del creador el suyo propio, entendiéndolo desde otro lugar y generando nuevos significados y perspectivas. En este sentido, seguiríamos la línea de la propuesta de Sanders (2006), quien también pone a artistas escénicos y performativos como ejemplos a seguir en IBA por desafiar las formas y por su transdisciplinariedad. Asimismo buscamos también el aprendizaje del cuestionamiento que estos artistas hacen del arte y de la realidad (Figura 1). 


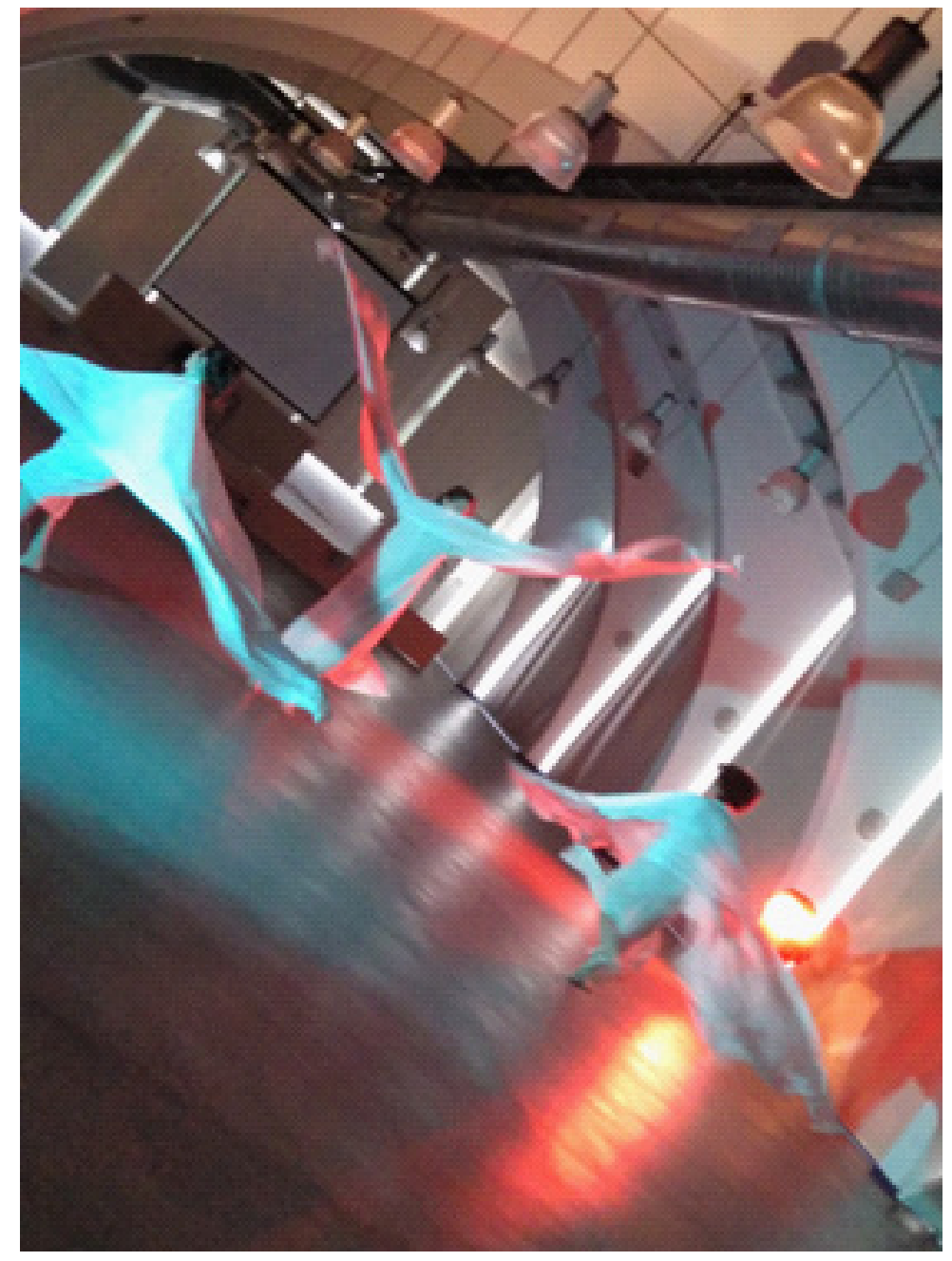

Figura 1. Puesta en escena basada en La danse serpentine, de Loïe Fuller (1890). Foto de la autora

Por último, y en el marco de una asignatura que de nuevo da cuenta de la perspectiva transdisciplinar que manejamos en el centro, describiremos un ejercicio de traducción inverso al propuesto para Literatura Dramática I. La asignatura es Dramaturgia Audiovisual (GAE3), en la que se dota en una primera parte a los/ las estudiantes de conocimientos sobre la historia y la teoría del cine, antes de entrar a aprender cómo desarrollar un guion cinematográfico. El ejercicio consiste en una aproximación a lo cinematográfico desde lo teatral. La idea del ejercicio es aprovechar esta mirada doble, teatral y cinematográfica, proponiéndoles que por parejas o tríos elijan una escena de una película de un listado propuesto. De nuevo, en una primera fase, realizan un estudio y análisis de la película, en este caso, desde el punto de vista fílmico, así como una profundización en la escena elegida, atendiendo al conflicto de la escena y de los personajes. Sin modificar el guion, trasladan la sintaxis cinematográfica (en 2D) a una perspectiva escénica (3D), convirtiendo una secuencia o conjunto de secuencias cinematográficas en una escena teatral, de modo que los diferentes movimientos de cámara, el montaje, 
MARTÍNEZ-LÓPEZ / Una experiencia encarnada de investigación escénica en estudios teatrales universitarios

etc. son traducidos al uso escénico del espacio y del tiempo. Simultáneamente se familiarizan con las diferencias entre interpretación para cine y para teatro, y con las percepciones que como espectadores tenemos de un formato y otro ${ }^{6}$ (Figura 2).

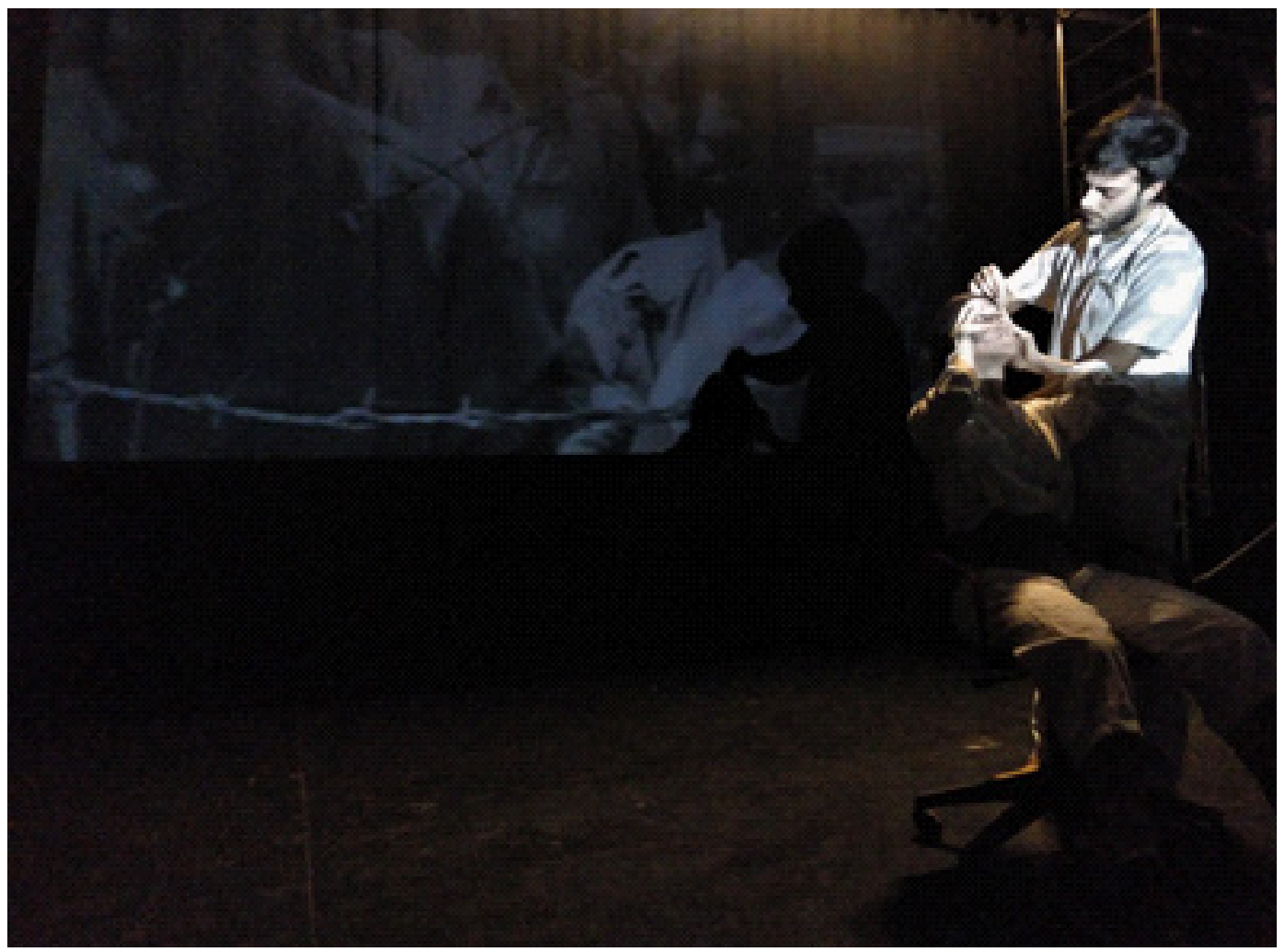

Figura 2. Adaptación escénica de La naranja mecánica, de Stanley Kubrick (1971). Foto de la autora

\section{Valores y criterios para la evaluación}

De entre todos los debates que hay en torno a la $\mathrm{ABR}$, uno de los más controvertidos es el de la evaluación. Leavy (2018b), cita a Faulkner para defender una serie de "criterios flexibles" (p. 575) generales interconectados, que podrían ser adaptados en función de la disciplina artística y de la naturaleza de cada proyecto. Para Borgdorff (2012), habría que valorar "el criterio de originalidad, la contribución al conocimiento y al entendimiento, la cuestión investigadora, el contexto de la investigación, los métodos de investigación, y la documentación y la diseminación de los hallazgos" (p. 208).

Los criterios de evaluación de la EU ERAM (UdG), sobre los que se apoya el tribunal de TFGs y en los que se basan los criterios de evaluación de cada asignatura se sustentan en tres pilares: idea, herramienta y expresión. Los subcriterios de estos,

6. Este último ejercicio tuve también la ocasión de ponerlo en práctica con estudiantes de segundo curso de la Licence en Arts du Spectacle de la Universidad París 8 en mayo de 2019, con motivo de una movilidad Erasmus de profesorado. 
perfilados y complementados por algunos de los propuestos por Leavy (2018b) y matizados por el planteamiento general de Borgdorff (2012) son los que se han seguido para valorar las prácticas anteriormente explicadas. Así:

Idea. Corresponde a la parte teórica. Comprende:

- Investigación. Tendría que ver con la metodología, y con la coherencia en el discurso.

- Creatividad. Tanto teórica como práctica. Relacionada con la innovación metodológica y las aportaciones del estudiante al objeto de estudio. Se valoran la autenticidad y la honestidad.

Herramienta. Planificación y realización del proyecto práctico, además de su descripción por escrito. Se valora la coherencia con la idea y los referentes, y la justificación de las elecciones creativas. También el trabajo en grupo.

Expresión. Presentación del trabajo práctico, en nuestro caso, la mayoría work-inprogress. Se valoran la calidad creativa, artística y ética, y la innovación.

Autoevaluación. (Por escrito). Se valora la mirada crítica del/de la estudiante o del grupo sobre su propio trabajo, teniendo siempre en cuenta la idea de cómo se sienten dentro de la práctica.

Reflexión colectiva. Finalmente, contrastando impresiones mediante el espacio abierto al diálogo tras la presentación de los trabajos, se evalúa la capacidad reflexiva de la obra, de generar múltiples significados y feedbacks por parte de la audiencia (Leavy, 2018) (en este caso, los compañeros).

Todos estos criterios podrían también agruparse en las categorías que propone Norris (cit. en Leavy, 2018b: 576) y cuya perspectiva resulta muy interesante: pedagogía, política, poiesis y posicionamiento público.

\section{Conclusiones}

A través de los ejercicios presentados en el bloque anterior -que parten de unos parámetros, una pauta, pregunta o hipótesis, como un trabajo de investigación-, hemos visto cómo se invita a los/las estudiantes a realizar un viaje que, debido a los métodos utilizados, no es lineal. Cada proceso es diferente, pero todos ellos son transformadores, y precisamente lo que se busca con ellos es abrirse a una multiplicidad de significados, desatando la reflexión individual y colectiva durante su realización y después mediante la experiencia compartida y dialógica con los compañeros-espectadores. En este viaje investigador y en este puzle que es la creación escénica contemporánea, el a/r/tógrafo, una vez desplegado el contexto de trabajo, ha de estar atento a cómo funcionan su pensamiento y emociones y las del alumnado, permitiendo que el proceso sea un lugar donde puedan aflorar talentos, dificultades en el aprendizaje, bloqueos, etc. cuestionándolo de manera constante, adaptándolo a cada grupo y haciéndolo evolucionar, igual que en un proceso creativo. En última instancia tendrá que valorar los ejercicios de una manera 
expandida, teniendo en cuenta tanto el objeto artístico, como el proceso, como el contexto de recepción del mismo.

Debemos aprovechar las pocas universidades que imparten estudios teatrales superiores no solo como espacios para enseñar una técnica o unos conocimientos teóricos, sino como generadores de contextos de creación e investigación donde aunar práctica artística, reflexión y discurso, y como puentes entre la tradición y la contemporaneidad, con sus redes y sus contextos de trabajo, como apuntan Sánchez y Pérez Royo (2010); utilizando además una mirada transversal. Ese ha sido el objetivo que se ha buscado con las prácticas propuestas y descritas, intentando enseñar al alumnado del Grado de Artes Escénicas de la Universitat de Girona a ser al mismo tiempo artistas escénicos e investigadores en el marco de una experiencia encarnada. Así, si partimos de la idea de que cualquier creador contemporáneo es también un investigador, podemos decir que estamos formando artistas investigadores que se sumergen además en la exploración de la historia desde el ancla de su presente.

Finalmente, analizado el marco teórico actual y habiendo experimentado y relatado nuevas posibles vías (conceptuales y prácticas) en relación al tema propuesto, podemos afirmar que aplicar a la pedagogía universitaria un proceso de investigación creativo y encarnado que va de la experiencia física y emocional al discurso, pero también a la inversa, puede considerarse un camino totalmente legítimo y aún por explorar en unos estudios cuyo campo teórico está también en plena reformulación, huyendo de antiguos parámetros. Gracias a esta perspectiva, podremos vencer "la brecha siempre presente entre las palabras y la visión", (Berger, 2017, p. 7), y bucear entre infinitas conexiones para que aparezca en los intersticios lo que no se puede decir con palabras; de la misma manera, la escritura de este artículo solo puede aproximarse en parte a la experiencia real de la vivencia de la práctica.

\section{Referencias}

Artaud, A. (2011). El teatro y su doble. Barcelona: Edhasa.

Austin, J. L. (2016). Cómo hacer cosas con palabras. Barcelona: Paidós.

Barone, T y Eisner, E. (2011). Arts Based Research. Los Ángeles: Sage.

Beck, J. L., Belliveau, G. Lea, G. W., y Wager, A. (2011). Delineating a Spectrum of Research-Based Theatre. En Qualitative Inquiry 17(8). Recuperado de: http://qix. sagepub.com/content/17/8/687.

Belliveau, G. y Lea, G. W. (Eds.) (2016). Research-based Theatre: An Artistic Methodology. Bristol: Intellect Ltd. 
Berger, J. (2017). Modos de ver. Barcelona: Editorial Gustavo Gili.

Boal, A. (2009). Teatro del oprimido. Barcelona: Alba Editorial.

Borgdorff, H (2012). The Conflict of the Faculties. Perspectives on Artistic Research and Academia. Leiden: Leiden University Press.

Bourriaud, N. (2015). Estética relacional. Buenos Aires: Adriana Hidalgo Editora.

Brook, P. (2015). El espacio vacío. Barcelona: Península

Chéjov, M. (1999). Sobre la técnica de la actuación inspirada. Barcelona: Alba Editorial.

De Botton, A. y Armstrong, J. (2017). El arte como terapia. Barcelona: Phaidon.

Della Fonte, L., Krasmanski, O., y Proz, J. (2015). Investigación en las artes visuales: la producción artística como investigación. Apuntes y reflexiones. En VV. AA. La investigación disciplinaria en lenguajes: cuadernos de investigación (pp. 7-25). Buenos Aires: Universidad Nacional de las Artes.

Dewey, J. (2008). El arte como experiencia. Barcelona: Paidós.

Eisner, E. W. (2004). El arte y la creación de la mente. Barcelona: Paidós.

Fischer-Lichte, E. (2017). Estética de lo performativo. Madrid: Abada Editores.

Hearing, T. y Jones, K. (2018). Film as Research/Research as Film. En En Leavy, P. (ed.). Handbook of Art-Based Research (pp. 425-436). New York \& London: The Guilford Press.

Hernández, F. (2008). La investigación basada en las artes. Propuestas para repensar la investigación en educación. En Educatio Siglo XXI, (26), 85-118.

Irwin, R.L., LeBlanc, N., Ryu, J. Y., y Belliveau, G. (2018). A/r/tography as Living Inquiry. En Lea, G. W., Belliveau, G., Wager, A., y Beck, J.L. (2011). A Loud Silence: Working with Research-based Theatre and A/R/Tography. International Journal of Education \& the Arts, 12(16). Recuperado de http://ijea.org/v12n16

Leavy, P. (2018a). Introduction to Arts-Based Research. En Leavy, P. (Ed), Handbook of Art-Based Research (pp. 3-21). New York \& London: The Guilford Press.

Leavy, P. (2018b). Criteria for evaluating Arts-Based Research. En Leavy, P. (Ed), Handbook of Art-Based Research (pp. 575-586). New York \& London: The Guilford Press.

Lehman, H.T. (2013). Teatro posdramático. Murcia: CENDEAC. 
MARTÍNEZ-LÓPEZ / Una experiencia encarnada de investigación escénica en estudios teatrales universitarios

Merleau-Ponty, M. (2017). El ojo y el espíritu. Madrid: Trotta.

Nietzsche, F. (2012). El nacimiento de la tragedia. Madrid: Alianza Editorial.

Norris, J. (2018). Reflections on the Techniques and Tones of Playbuilding by a Director/Actor/Researcher/Teacher. En Leavy, P. (Ed.), Handbook of Art-Based Research (pp. 288-307). New York \& London: The Guilford Press.

Sánchez, J. A. (1999). La escena moderna. Manifiestos y textos sobre teatro en la época de las vanguardias. Madrid: Akal.

Sánchez, J. A. y Pérez Royo, V. (2010). La investigación en artes escénicas. Introducción. En Cairon, 13. Revista de estudios de danza. Práctica e investigación, 5-15. Alcalá de Henares: Universidad de Alcalá.

Saldaña, J. (2011). Ethnotheatre: Research from page to stage. California: Left Coast Press, 2011.

Sanders, J. H. (2006). Performing Arts-Based Education Research: An Epic Drama of Practice, Precursors Problems and Possibilities. Studies in Art Education. A Journal of Issues and Research 2006, 48(1), 89-107. Recuperado de: https://www. jstor.org/stable/25475807?seq=1\#page_scan_tab_contents

Snowber, C. (2018). Living, Moving, and Dancing: Embodied Ways of Inquire. En Leavy, P. (Ed.), Handbook of Art-Based Research (pp. 247-266). New York \& London: The Guilford Press.

Sullivan, G. (2004). Art Practice Research Inquiry in the Visual Arts. New York: Teachers College Columbia University.

Szondi, P. (2011). Teoría del drama moderno (1880-1905). Una tentativa sobre lo trágico. Madrid: Dykinson.

Vidiella, J. (2018). El arte de la investigación o de la investigación basada en las artes. En VV. AA., Polaritats 2016-2017. Un año de mediación artística (pp. 6075). Barcelona: Homesession i Institut Ramon Llul. 